\title{
Deep Venous Thrombosis and Agenesis of the Inferior Vena Cava: Typical Presentation Infrequent Anomaly
}

\author{
Fernanda Castro L, ${ }^{1 *}$ Álvaro Zapico, ${ }^{2}$ Ricardo Wilhelm ${ }^{3}$ \\ ${ }^{1}$ General Surgeon, Surgery Department of San Martin de Quillota Hospital, Chile \\ ${ }^{2}$ Vascular Surgeon, Surgery Department of San Martin de Quillota Hospital, Chile \\ ${ }^{3}$ Radiologist, Radiology Department of San Martin de Quillota Hospital, Chile
}

\begin{abstract}
Abnormalities of the inferior vena cava (IVC) are rare. Its embryological development occurs between the sixth and eighth week of gestation, and depends on the persistence or regression of 3 pairs of veins: the posterior cardinal veins, the sub cardinal veins and the supra cardinal veins. The type of congenital alteration depends the moment that embryogenesis is altered. The most frequent clinical presentation is deep vein thrombosis, which occurs mainly in young men. Other clinical presentations are pelvic venous congestion and chronic venous insufficiency. The diagnosis requires a high diagnostic suspicion, and it is mainly through contrast-enhanced computed tomography of the abdomen and pelvis. The treatment will depend on the type of malformation and its presentation.
\end{abstract}

Keywords: Upper extremity deep vein thrombosis, Vena cava inferior, Congenital abnormalities

\section{Introduction}

Congenital anomalies in the inferior vena cava (IVC) are rare, with its agenesis being the less frequent manifestation, with an approximate incidence of $0.005 \%$ to $1 \%$ in the general population. ${ }^{1,2}$ Other, more common anomalies are chronic venous insufficiency, pelvic congestion syndrome and deep vein thrombosis. ${ }^{1-6}$ Additional less frequent symptoms are described, such as lumbar pain, secondary to nerve compression caused by the development of collaterals. ${ }^{1,5}$ However, generally this pathology is completely asymptomatic. In this publication, we will describe the clinical case of a DVT associated to an uncommon anomaly of the IVC and we will discuss the available evidence regarding the association of these pathologies.

\section{Clinical Case}

16-year-old male with no previous morbidity in his clinical records, arrived at the Emergency Room seeking treatment for a two- week long case of pain and swelling in his lower right extremity, with no other associated symptomology. The patient had no smoking habit, had not undergone prior surgeries, had not traveled long distances, nor had he suffered trauma within the prior 30 days. In the physical exam, the increase in volume of the lower right extremity was noted and a positive Homan's sign. The rest of the physical exam produced no other pathological findings. Due to suspicion of a deep vein thrombosis a Venous Doppler ultrasound was performed, which confirmed the diagnosis of a femoral-popliteal DVT. As part of the etiopathogenic study, a contrast-enhanced ultrasound of the pelvis and abdomen was done, on which the agenesis of the suprarenal inferior vena cava was identified, as well as multiple resulting collaterals (Figure 1). Negative thrombophilia study. An echocardiogram was taken to complete the check for associated malformations, with no pathological findings. The decision was made to treat the patient with graduated compression stockings associated to low-molecular weight heparin, with later overlap to vitamin $\mathrm{K}$ an-

\begin{tabular}{|l|l|}
\hline \hline Quick Response Code: & *Corresponding author: Fernanda Castro Leiva, Surgery Department of San Martin de Quillo- \\
ta Hospital, Quillota, Chile \\
Received: 08 June, 2021 Published: 28 July, 2021 \\
Citation: Fernanda Castro FL, Zapico A, Wilhelm R. Deep Venous Thrombosis and Agenesis \\
of the Inferior Vena Cava: Typical Presentation Infrequent Anomaly. Curr Inv Cln Med Res. \\
2021;1(2):1-4. DOI: 10.53902/CICMR.2021.01.000510
\end{tabular}


tagonists and the indefinite extension of the treatment. There was a good clinical response, with a gradual decrease of the edema. In the 18-month follow-up, the patient remains asymptomatic. There have been no new episodes of DVT, nor signs of post-thrombotic syndrome.

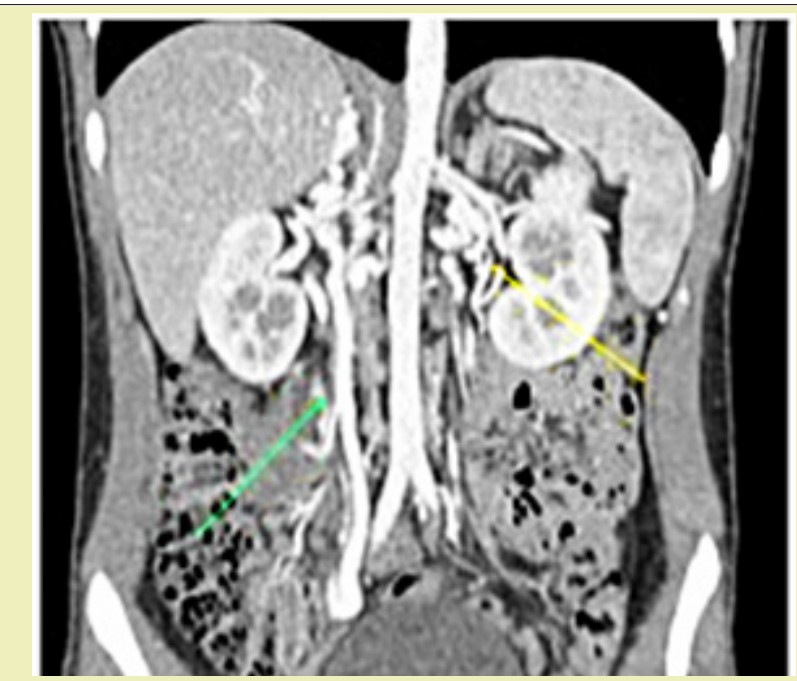

Figure 1: Contrast Enhanced CT with coronal reconstruction. Absence of IVC in its supra and infrarenal segments. Development of multiple paravertebral collaterals (yellow arrows). Dilated right testicular veins (green arrow).

\section{Discussion}

Congenital anomalies in the inferior vena cava (IVC) are rare, with agenesis being the less frequent manifestation, with an approximate incidence of $0.005 \%$ to $1 \%$ in the general population. ${ }^{1,2}$ However, in young patients with a deep vein thrombosis diagnosis, the incidence increases to $5 \%{ }^{5,6}$ Anomalies in the development of the inferior vena cava. The embryological development of the inferior vena cava takes places between the sixth and eight week of gestation, and depends on the persistence or regression of 3 pairs of veins: the posterior cardinal veins, the sub cardinal veins and the supra-cardinal vein (Figure 2). ${ }^{1,7-10}$ The type of anomaly varies according to the moment of embryological development that presents an alteration, and so the presence of a left IVC or double IVC is owed to the persistence of the left supra-cardinal vein, accompanied or not by the atrophy of the right supra-cardinal vein., ${ }^{7,9}$ While the anomalies of the renal vein are caused by the persistence of the inter-supra cardinal associated or not to the regression of the inter-sub cardinal vein, manifesting during adult life as a retro aortic left renal vein or a circumarctic left renal vein. ${ }^{7,9}$ Regarding the absence of IVC, there is no clarity as to its etiology. Some authors have proposed a secondary origin of the thrombosis of the intrauterine IVC, resulting in its agenesis and the redistribution of the venous return into four different paths: the deep collaterals (paravertebral and periutheral), middle collaterals (gonadal), superficial collaterals (epigastric, internal mammary and subclavian) and via the portal system through the hemorrhoidal veins.
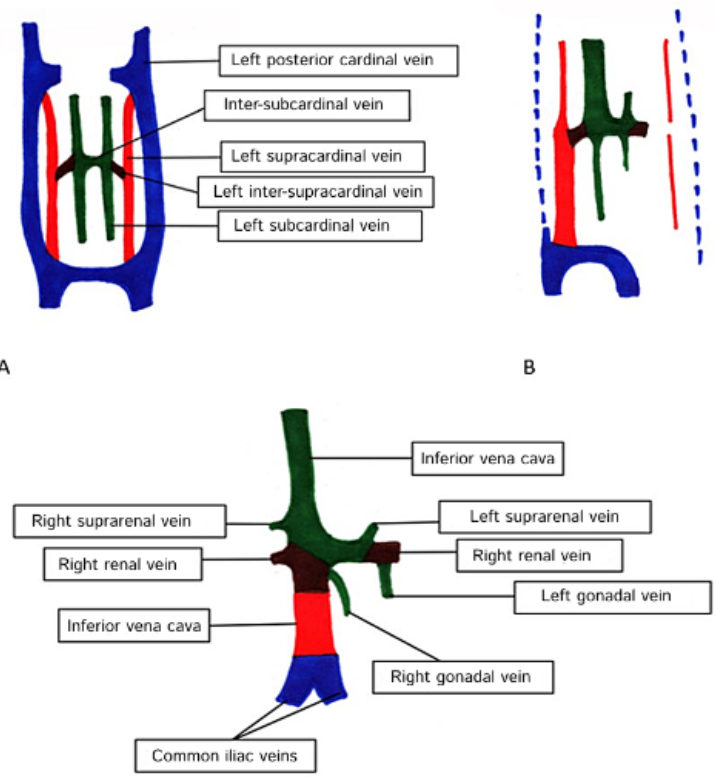

\section{Contar palabras}

\section{Estadísticas:}

Páginas

Palabras

Caracteres (sin espacios)

Caracteres (con espacios)

9.906

Párrafos

Líneas

Incluir cuadros de texto, notas al pie y notas al final

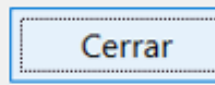

Figure 2: The embryological development of the IVC depends on the persistence or regression of 3 pairs of veins, the posterior cardinal veins (blue), the sub cardinal veins (green) and the supra cardinal veins (red).

A. Posterior cardinal veins are dominant in the sixth week of gestation. By the seventh week, sub cardinal veins become predominant. In the eighth week, supracardinal veins are predominant and the formation of the inter-supracardinal veins (retroaortic) and intersubcardinal (preaortic) veins is complete.

B. The regression of the left veins and the right posterior cardinal vein.

C. Finally, the IVC is formed by the right sub cardinal vein, and in its infrarenal portion by the right supracardinal vein. The renal veins are developed from the inter-supra cardinal veins.

\section{Clinical Presentation}

The inadequate blood flow return caused by the alteration of the IVC causes vein hypertension, despite the development of a considerable collateral supply, leading to vein stasis that can result in 
thrombosis. ${ }^{5,6,11}$ The presence of pulmonary embolism in DVT patients and IVC atresia is rare, ${ }^{6,11}$ probably because the migration of thrombus is prevented by the wide network of compensatory collateral circulation. ${ }^{11}$ The absence of IVC can also manifest as chronic vein insufficiency and pelvic congestion syndrome. ${ }^{1-5,11}$ However, most patients with IVC agenesis are asymptomatic and diagnosis is incidental. ${ }^{5}$ Patients that present deep vein thrombosis in the presence of IVC alterations usually present lumbar and/or abdominal pain, associated to lower extremity edema. ${ }^{12}$ Thrombosis usually affects distal segments, such as the IVC or iliac veins, more frequently in a bilateral manner. ${ }^{4-6}$ Risk factors that can trigger a prothrombotic state in patients with IVC anomalies are prolonged immobilization, trauma, infections, neoplasia, surgeries, pregnancies and the use of prothrombotic medication, which is why it becomes important to record these elements in the medical history of patients that treated for deep vein thrombosis. ${ }^{12}$ Furthermore, numerous reports have described the association of intense exercise and deep vein thrombosis in patients with congenital IVC anomalies. ${ }^{13}$

\section{Diagnosis and Complementary Studies}

An ultrasound is usually the first imaging modality in the evaluation of patients with deep vein thrombosis. ${ }^{6}$ However, inferior vena cava anomalies may fail to be diagnosed during this study, making it essential to complete the etiologic study of young or lowrisk patients of deep vein thrombosis with a contrast-enhanced ultrasound of the pelvis and abdomen to establish the existence of IVC anomalies, as well as other associated risk factors. ${ }^{6,14}$ Other options for diagnostic study of IVC are the nuclear magnetic resonance as well as phlebography, although these options are currently less common. Bibliographic revisions describe the concomitant presence of thrombophilia in up to $40 \%$ of patients with IVC alterations that present with DVT. ${ }^{5,12,15}$ Defining the existence of this link is fundamental in order to define the long-term handling of these patients. For this reason, the study of these patients must always include screening for thrombophilia. Furthermore, the IVC alterations may be associated with other malformations such as situs inversus, dextrocardia, polysplenia or asplenia. In the case of IVC agenesis, this may be associated to agenesis or renal hypoplasia, constituting KILT syndrome, ${ }^{1,3}$ so studies intended to rule out the presence of these associations is advised.

\section{Treatment}

The treatment of inferior vena cava malformations will depend on the type of alterations and its clinical presentation. In the case of deep vein thrombosis in patients with IVC agenesis, the use of compression or antithrombotic stockings, together with oral anticoagulants is considered today to be the best approach, ${ }^{1,2,6}$ associated to minimizing the factors that increase the risk of DVT. ${ }^{3}$ The duration of the anticoagulant treatment is under debate and there is no clear scientific evidence to support a definitive answer. Some authors recommend maintaining it for 3 months in cases where there is no coexistent thrombophilia. ${ }^{3,6}$ Another approach is to maintain the treatment on the long term, in order to decrease the reoccurrence of DVT and the incidence of post thrombotic syndrome. ${ }^{5,12}$ There are publications that suggest that catheter-directed thrombolysis could be advised as the treatment of choice for acute iliofemoral DVT in patients with IVC atresia, since this treatment may achieve satisfactory clinical results, including the permeability of the deep veins. ${ }^{5,12}$ However, there are insufficient studies to confirm the benefits of this therapy versus the conservative treatment with anticoagulants, in patients with associated deep vein thrombosis and agenesis of the IVC. There has also been reports of surgical interventions such as the venous thrombectomy or even the prosthetic replacement of the IVC, with successful results. The surgical approach is only recommended for those patients with rapid deterioration of the chronic venous insufficiency, or those that present DVT and concomitant collapse of the collateral venous drainage. . $^{1,3,6,16}$

\section{Conclusion}

The agenesis of the inferior vena cava is a rare alteration, which must be suspected in young patients with deep vein thrombosis that present no other evident causes, since it is this group that presents a higher incidence rate. The preferred methods of study are the venous Doppler ultrasound and the contrast-enhanced tomography. The etiopathogenic study must always include the search for associated malformations and the study of thrombophilia's, due to its frequent association and importance to the long-term approach. Due to the rare nature of this pathology, there are no randomized clinical studies that compare its different treatment alternatives. However, existing evidence, though limited to retrospective case reports, suggest the efficiency and safety of treatment with anticoagulants. Aspects such as the duration of treatment with anticoagulants are still controversial in the absence of thrombophilia's. Evidence suggests a lesser incidence of post thrombotic syndrome when a catheter-directed thrombolysis is performed in comparison to exclusive use of anticoagulants; however, further randomized prospective studies are needed. More aggressive therapeutic methods, such as surgery, are an alternative for serious cases or for those cases that fail to respond to more conservative treatments.

\section{Acknowledgments}

None.

\section{Funding}

None.

\section{Conflicts of Interest}

Authors declare that there is no conflict of interest. 


\section{References}

1. Morosetti D, Picchi E, Calcagni A, et al. Anomalous development of the inferior vena cava: Case reports of agenesis and hypoplasia. Radiology Case Reports. 2018;13(4):895-903.

2. Leitão A, Esteves J, Abreu J, et al. Deep Venous Thrombosis and a Very Rare Finding: Inferior Vena Cava Infra-renal Segment Agenesis. European Journal of Case Reports in Internal Medicine. 2019;6(3):1.

3. Ramos Aranda J, Ramírez Cerda C, Cohen Mussali S, et al. Inferior Vena Cava Agenesis: An Unusual Cause of Deep Vein Thrombosis and Pulmonary Embolism in Young Adult Patients. EJVES Short Reports. 2018;39:12-15.

4. Langer F, Dos Santos D, Suertegaray G, et al. Bilateral Deep Vein Thrombosis Associated with Inferior Vena Cava Agenesis in a Young Patient Manifesting as Low Back Pain. Acta Médica Portuguesa. 2017;30(4):333.

5. Broholm R, Jørgensen M, Just S, et al. Acute Iliofemoral Venous Thrombosis in Patients with Atresia of the Inferior Vena Cava Can Be Treated Successfully with Catheter-directed Thrombolysis. Journal of Vascular And Interventional Radiology. 2011;22(6):801-805.

6. G Gayer, J Luboshitz, M Hertz, et al. Congenital Anomalies of the Inferior Vena Cava Revealed on CT in Patients with Deep Vein Thrombosis. American Journal of Roentgenology. 2003;180:3729-732.

7. González J, Gaynor J, Albéniz L, et al. Inferior Vena Cava System Anomalies: Surgical Implications. Current Urology Reports. 2017;18(2).

8. Shin D, Sandstrom C, Ingraham C, et al. The inferior vena cava: a pictorial review of embryology, anatomy, pathology, and interventions. Abdominal Radiology. 2019;44(7):2511-2527.
9. Morato A, Quiroz O, Ramírez J. Anomalías congénitas de la vena cava inferior. El rol de la tomografía computada multidetector. Anales de Radiología México. 2012;2:104-113.

10. Motta Ramírez G, Mundo Gómez C, Ramírez Arias J. La vena cava y sus variantes anatómicas. Revista mexicana de angiología. 2010;38:19-29.

11. Khalid M, Nukavarapu M, Shah R, et al. Rare case of a kidney and inferior vena cava abnormalities with extensive lower extremity deep vein thrombosis in a young healthy male. Clinics and Practice. 2018.

12. Pozzi A, El Lakis MA, Chamieh J, et al. The Typical Presentation Spectrum of Deep Vein Thrombosis Associated with Inferior Vena Cava Malformations. Thrombosis. 2016;2016:4965458.

13. García Fuster M, Forner M, Flor Lorente B, et al. Inferior Vena Cava Malformations and Deep Venous Thrombosis. Revista Española de Cardiología. 2006.

14. Lambert M, Marboeuf P, Midulla M, et al. Inferior vena cava agenesis and deep vein thrombosis: 10 patients and review of the literature. Vascular Medicine. 2010;15(6):451-459.

15. Sagban T, Scharf R, Wagenhäuser M, et al. Elevated risk of thrombophilia in agenesis of the vena cava as a factor for deep vein thrombosis. Orphanet J Rare Dis. 2015.

16. Menezes T, Haider E, Al Douri F, et al. Pelvic congestion syndrome due to agenesis of the infrarenal inferior vena cava. Radiology Case Reports. 2019;14(1):36-40. 